\title{
Formation of supermassive black hole binaries and massive seed SMBHs in gas-rich mergers
}

\author{
Mayer, L
}

\begin{abstract}
We review the results of multi-scale, hydrodynamical simulations of major mergers between galaxies with or without central supermassive black holes (SMBHs) to investigate the orbital decay of $\mathrm{SMBH}$ pairs in galactic nuclei and the formation of massive SMBH seeds via direct gas collapse. Both SPH simulations and AMR simulations are carried out. The complex balance between heating and cooling is modeled via an effective EOS with varying adiabatic index apparopriate for the conditions of an intense nuclear starburst such as that expected during and after the merger. Prominent gas inflows due to tidal torques produce nuclear disks at the centers of merger remnants whose properties depend sensitively on the details of gas thermodynamics. In parsec-scale resolution simulations starting with two SMBHs originally at the centers of the two galaxies, a SMBH binary forms very rapidly, less than a million years after the merger of the two galaxies, owing to the drag exerted by the surrounding gaseous nuclear disk. Binary formation is significantly suppressed if heating, by e.g. radiative feedback from the accreting SMBHs, renders cooling negligible. The nuclear disk rearranges its mass distribution in response to a second, internal gas inflow occurring while the binary sinks. The inflow is driven by spiral instabilities imprinted by the final collision between the two galactic cores. In simulations with 0.1 pc resolution, the gas inflow continues all the way down to the center and peaks at $>104$ Msolar/yr, producing a Jeans-unstable supermassive central cloud (with mass a few times 108 Msolar) only $105 \mathrm{yr}$ after the merger. If the collapse continues, the cloud could form a massive black hole seed (Mseed $>105$ Msolar) after prior formation of a supermassive star or quasi-star. The massive SMBH seed can grow up to a billion solar masses in less than a billion years by accreting the surrounding nuclear gas. If the gas-rich merger occurs at $z>8$, this is then a new, attractive way to explain the rapid emergence of the bright QSOs discovered by the Sloan Digital Sky survey at $z>6$, which does not require the assumption of primordial gas composition in order to suppress cooling below $104 \mathrm{~K}$ and star formationas in models starting from unstable, isolated protogalactic disks. If there is a pre-existing pair of SMBHs their orbital decay stalls at parsec scales because, as a result of the formation of the supermassive cloud, the nuclear disk density decreases outside the cloud, yielding much weaker dynamical friction. We envision a new scenario in which direct formation of massive black hole seeds and SMBH binary formation are mutually exclusive; if a SMBH is already present in the nuclear disk it can stabilize it and weaken the secondary inflow via its energetic feedback, maintaining a high enough density where the SMBHs are located and assisting their sinking.
\end{abstract}

DOI: https://doi.org/10.1063/1.3458484

Posted at the Zurich Open Repository and Archive, University of Zurich

ZORA URL: https://doi.org/10.5167/uzh-41704

Conference or Workshop Item

Published Version 
Originally published at:

Mayer, L (2009). Formation of supermassive black hole binaries and massive seed SMBHs in gas-rich mergers. In: Hunting for the Dark: the Hidden Side of Galaxy Formation, Qawra, Malta, 19 October 2009 - 23 October 2009. American Institute of Physics, 181-194.

DOI: https://doi.org/10.1063/1.3458484 


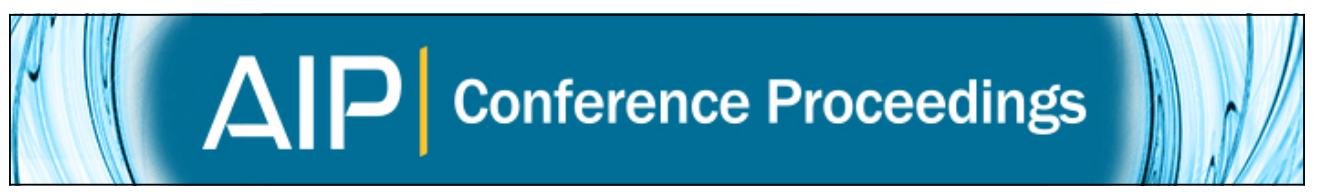

\section{Formation of Supermassive Black Hole Binaries and Massive Seed SMBHs in Gas-Rich Mergers}

Lucio Mayer

Citation: AIP Conference Proceedings 1240, 181 (2010); doi: 10.1063/1.3458484

View online: http://dx.doi.org/10.1063/1.3458484

View Table of Contents:

http://scitation.aip.org/content/aip/proceeding/aipcp/1240?ver=pdfcov

Published by the AIP Publishing

Articles you may be interested in

Black Hole Binary Mergers Within Gas Discs

AIP Conf. Proc. 1240, 231 (2010); 10.1063/1.3458494

On the inspiral of massive black holes in gas-rich galaxy mergers

AIP Conf. Proc. 924, 705 (2007); 10.1063/1.2774931

Gravitational Radiation From Super-Massive Black-Hole Binaries

AIP Conf. Proc. 686, 191 (2003); 10.1063/1.1629431

Observational evidence for supermassive black hole binaries

AIP Conf. Proc. 686, 161 (2003); 10.1063/1.1629428

Massive Black Hole Growth and Formation

AIP Conf. Proc. 686, 141 (2003); 10.1063/1.1629426 


\title{
Formation of Supermassive Black Hole Binaries and Massive Seed SMBHs in Gas-Rich Mergers
}

\author{
Lucio Mayer \\ Institute for Theoretical Physics, University of Zürich, Switzerland
}

\begin{abstract}
We review the results of multi-scale, hydrodynamical simulations of major mergers between galaxies with or without central supermassive black holes (SMBHs) to investigate the orbital decay of SMBH pairs in galactic nuclei and the formation of massive SMBH seeds via direct gas collapse. Both SPH simulations and AMR simulations are carried out. The complex balance between heating and cooling is modeled via an effective EOS with varying adiabatic index $\gamma$ apparopriate for the conditions of an intense nuclear starburst such as that expected during and after the merger. Prominent gas inflows due to tidal torques produce nuclear disks at the centers of merger remnants whose properties depend sensitively on the details of gas thermodynamics. In parsec-scale resolution simulations starting with two SMBHs originally at the centers of the two galaxies, a SMBH binary forms very rapidly, less than a million years after the merger of the two galaxies, owing to the drag exerted by the surrounding gaseous nuclear disk. Binary formation is significantly suppressed if heating, by e.g. radiative feedback from the accreting SMBHs, renders cooling negligible.

The nuclear disk rearranges its mass distribution in response to a second, internal gas inflow occurring while the binary sinks. The inflow is driven by spiral instabilities imprinted by the final collision between the two galactic cores. In simulations with $0.1 \mathrm{pc}$ resolution, the gas inflow continues all the way down to the center and peaks at $>10^{4} M_{\odot} / y r$, producing a Jeans-unstable supermassive central cloud (with mass a few times $10^{8} M_{\odot}$ ) only $10^{5} \mathrm{yr}$ after the merger. If the collapse continues, the cloud could form a massive black hole seed $\left(M_{\text {seed }}>10^{5} M_{\odot}\right)$ after prior formation of a supermassive star or quasi-star. The massive SMBH seed can grow up to a billion solar masses in less than a billion years by accreting the surrounding nuclear gas. If the gas-rich merger occurs at $z>8$, this is then a new, attractive way to explain the rapid emergence of the bright QSOs discovered by the Sloan Digital Sky survey at $z>6$, which does not require the assumption of primordial gas composition in order to suppress cooling below $10^{4} \mathrm{~K}$ and star formation as in models starting from unstable, isolated protogalactic disks. If there is a pre-existing pair of SMBHs their orbital decay stalls at parsec scales because, as a result of the formation of the supermassive cloud, the nuclear disk density decreases outside the cloud, yielding much weaker dynamical friction. We envision a new scenario in which direct formation of massive black hole seeds and SMBH binary formation are mutually exclusive; if a SMBH is already present in the nuclear disk it can stabilize it and weaken the secondary inflow via its energetic feedback, maintaining a high enough density where the SMBHs are located and assisting their sinking.
\end{abstract}

\section{INTRODUCTION}

In recent years, compelling dynamical evidence has indicated that supermassive black holes (SMBHs) are ubiquitous in galactic nuclei [e.g. 1]. According to the standard modern theory of cosmological structure formation, the Cold Dark Matter (CDM) paradigm [e.g. 2], galaxies in the Universe grow through a complex process of continuous mergers and agglomeration of smaller systems. Thus, if more than one of the protogalactic fragments contained a SMBH, the formation of SMBH binaries during galaxy assembly 
will be almost inevitable [e.g. 3].

In a purely stellar background, as the binary separation decays, the effectiveness of dynamical friction slowly declines, and the pair can become tightly bound via three-body interactions, namely by capturing stars that pass close to the black holes and ejecting them at much higher velocities [e.g. 4]. If the hardening continues to sufficiently small relative distances, gravitational wave $(\mathrm{GW})$ emission becomes the dominant source of orbital energy loss and the two SMBHs may coalesce in less than a Hubble time. In this case, the GW emission should be detected by the forthcoming gravitational wave interferometers, such as the planned Laser Inteferometer Space Antenna (LISA), becoming a novel and unique probe of both the fabric of space time and cosmological structure formation [e.g. 5]. However, the binary orbit may stop shrinking before gravitational radiation becomes relevant as there is a finite supply of stars on intersecting orbits [e.g. 6].

During the assembly of galaxies, especially at high $z$, their SMBHs likely evolve within gas-rich environments. Merging systems such as the Ultraluminous Infrared Galaxies (ULIRGs) NGC 6240 and Arp 220 harbor large concentrations of gas, in excess of $10^{9} M_{\odot}$, at their center, in the form of either a turbulent irregular structure or of a kinematically coherent, rotating disk [e.g. 7]. Massive rotating nuclear disks of molecular gas are also ubiquitous in galaxies that appear to have just undergone a major merger, such as Markarian 231 [8]. Gas dynamics may thus profoundly affect the pairing of SMBHs both during and after their host galaxies merge [e.g. 9, 10].

Recent simulations of the orbital evolution of SMBHs within an equilibrium, rotationally-supported, gaseous disk have shown that dynamical friction against the gaseous background leads to the formation of a tightly bound SMBH binary with a final separation of $<1 \mathrm{pc}$ in about $10^{7} \mathrm{yr}[11,12,13]$. Here we review the results of high-resolution $N$-body + smoothed particle hydrodynamics $(\mathrm{SPH})$ as well as adaptive mesh refinement (AMR) simulations of mergers between galaxies with central SMBHs having enough dynamic range to follow the black holes from hundreds of kiloparsecs down to sub-parsec scales, bridging more than ten orders of magnitude in density. Simulations by Cuadra, discussed in these proceedings, deal with a later stage of the decay, starting when the two SMBHs have already reached sub-pc scales.

The GW emission event rate depends not only on the efficiency of the binary coalescence process, but also on the mechanism of SMBH formation and growth, in particular on how massive are the typical merging SMBHs as a function of redshift, with respect to the LISA detection window (in the range $10^{4}-10^{7} M_{\odot}$ ), as discussed by [14]. Therefore, understanding the formation of SMBHs is pivotal in order to interpret future $\mathrm{GW}$ detections, and, even earlier, to make predictions on detection rates themselves. Furthermore, it is by itself a crucial aspect of our attempt to understand the co-evolution of galaxies and SMBHs. Observationally, an important constraint comes from the existence of billion solar mass SMBHs powering the bright QSOs discovered by the SDSS at $z>6$ [15]. A plausible formation model must explain naturally their rapid appearance. Instead, the conventional model, that relies on an early formation of light, hundred solar mass SMBH seeds at $z>15$ from the collapse of Pop III stars plus their subsequent growth via gas accretion [16], seems unable to explain them [17]. We will review recent work employing another set of galaxy merger simulations, this time reaching a spatial resolution of $0.1 \mathrm{pc}$, that lay the basis for a new rapid formation scenario of massive 
$\left(>10^{5} M_{\odot}\right)$ SMBH seeds - direct gas collapse in gas-rich galaxy mergers. As we will explain, these massive seeds can grow to billion solar masses fast enough to accomodate the observational evidence coming from the existence of the high-z SDSS QSOs.

\section{METHODS}

The aim of this study is to investigate the orbital evolution and pairing of SMBHs in multi-scale galaxy mergers in the hydrodynamical regime. A thorough description of our methods is presented in [10] and [18, hereafter M07] and we summarize them here. All the simulations presented in this paper start with two identical spiral galaxies, comprising a disk of stars and gas with an exponential surface density distribution, a spherical, non-rotating Hernquist bulge, and a spherical and isotropic NFW dark matter halo. We adopted parameters from the Milky Way model A1 of [19] to initialize the galaxy models.

Specifically, the dark matter halo had a virial mass of $M_{\mathrm{vir}}=10^{12} M_{\odot}$, a concentration parameter of $c=12$, and a dimensionless spin parameter of $\lambda=0.031$. The mass, thickness and resulting scale length of the disk were $M_{d}=0.04 M_{\mathrm{vir}}, z_{0}=0.1 R_{d}$, and $R_{d}=3.5 \mathrm{kpc}$, respectively. The bulge mass and scale radius were $M_{b}=0.008 M_{\mathrm{vir}}$ and $a=0.2 R_{d}$, respectively. The halo was adiabatically contracted to respond to the growth of the disk and bulge resulting in a model with a central total density slope close to isothermal. The galaxy models were consistent with the stellar mass Tully-Fisher and size-mass relations. A softened particle of mass $2.6 \times 10^{6} M_{\odot}$ was placed at the center of the bulge to represent a SMBH. This choice satisfies the $M_{\mathrm{BH}}-\sigma$ relation [10]. Lastly, the gas fraction, $f_{\mathrm{g}}$, was chosen to be $10 \%$ of the total disk mass. We used a standard cooling function for a primordial mixture of atomic hydrogen and helium. We also shut off radiative cooling at temperatures below $2 \times 10^{4} \mathrm{~K}$ that is a factor of $\sim 2$ higher than the temperature at which atomic radiative cooling would drop sharply due to the adopted cooling function. With this choice we effectively take into account non-thermal, turbulent pressure to model the warm ISM of a real galaxy.

The galaxies were placed on parabolic orbits with pericentric distances that were $20 \%$ of the halo virial radius $\left(r_{\text {peri }} \sim 50 \mathrm{kpc}\right.$ ), typical of cosmological mergers [e.g. 20]. The initial separation of the halo centers was twice their virial radii and their initial relative velocity was determined from the corresponding Keplerian orbit of two point masses. Each galaxy consisted of $10^{5}$ stellar disk particles, $10^{5}$ bulge particles, and $10^{6}$ dark matter particles. The gas component was represented by $10^{5}$ particles. We employed a gravitational softening of $\varepsilon=100 \mathrm{pc}$ for both the dark matter and baryonic particles of the galaxy, and $\varepsilon=30 \mathrm{pc}$ for the particle representing the SMBH.

During the interaction between the two galaxies, the relative separation of the black holes followed that of the galactic cores in which they were embedded. The merging galaxies approached each other several times as they sank into one another via dynamical friction. After $\sim 5$ Gyr, the dark matter halos had nearly merged and the two baryonic cores, separated by about $6 \mathrm{kpc}$, continued to spiral down. As much as $60 \%$ of the gas originally present in the galaxies was funneled to the inner few hundred parsecs of each core by tidal torques and shocks occurring in the repeated fly-bys between the two galaxies [e.g. 21]. Each SMBH was embedded in a rotating gaseous disk of mass 
$\sim 4 \times 10^{8} M_{\odot}$ and size of a few hundred parsecs which was produced by the gas inflow.

Just before the last pericentric passage of the two merging galaxies, we adopted the technique of particle splitting to increase the gas mass resolution in the central region of the computational volume. By selecting a large enough volume for the fine grained region one can avoid dealing with spurious effects at the coarse/fine boundary, such as two-body heating due to scattering by massive particles of the low-resolution region. We selected the volume of the fine-grained region to be large enough to quarantee that the dynamical timescales of the entire coarse-grained region were much longer than those corresponding to the refined region. Specifically, we performed the splitting in a volume of $30 \mathrm{kpc}$ in radius at the point where the two galaxy cores were separated by only $6 \mathrm{kpc}$. The new particles were randomly distributed according to the SPH smoothing kernel within a volume of size $\sim h_{p}^{3}$, where $h_{p}$ is the smoothing length of the parent particle. The velocities of the child particles were equal to those of their parent particle (ensuring momentum conservation) and so was their temperature, while each child particle was assigned a mass equal to $1 / N_{\text {split }}$ the mass of the parent particle, where $N_{\text {split }}$ is the number of child particles per parent particle. The mass resolution in the gas component was originally $2 \times 10^{4} M_{\odot}$ and became $\sim 3000 M_{\odot}$ after splitting, for a total of $\sim 1.5$ million SPH particles. For the standard calculations, the softening of the gas particles was set to 2 pc. We note that the local Jeans length was always resolved by 10 or more SPH smoothing kernels [e.g. 22] in the highest density regions of the refined simulations. The softening of the black holes was also reduced from $30 \mathrm{pc}$ to $2 \mathrm{pc}$ (or to $0.1 \mathrm{pc}$ in the highest resolution simulations), while the softening of dark matter and stellar particles remained $100 \mathrm{pc}$ as they were not split in order to limit the computational burden. Therefore, stellar and dark matter particles essentially provide a smooth background potential, while the computation focused on the gas component which dominates by mass the nuclear region. All simulations were performed with GASOLINE, a multistepping, parallel Tree-SPH $N$-body code [23].

The radiation physics in the refined simulations was modeled via an "effective" equation of state that accounts for the net balance of radiative heating and cooling. The value of the adiabatic index, $\gamma$, namely the ratio between the specific heats, is the parameter that controls the degree of dissipation in the gas. While the various cooling and heating mechanisms should be followed directly, this simple scheme allows us to investigate the effect of thermodynamics on the structure of the merger remnant and on the orbital decay of the black holes. Lastly, we tested that the transition between the two thermodynamic schemes used in the different parts of the simulation did not introduce spurious fluctuations in the hydrodynamical variables (M07).

\section{FORMATION OF SMBH BINARIES AND THE EFFECT OF THERMODYNAMICS}

Calculations that include radiative transfer show that the thermodynamic state of a solar metallicity gas heated by a starburst can be well approximated by an ideal gas with adiabatic index $\gamma=1.3-1.4$ over a wide range of densities [24]. For the standard refined simulation discussed in the present work, we adopted $\gamma=7 / 5$.

The gaseous cores finally merge at $t \sim 5.12 \mathrm{Gyr}$, forming a single nuclear disk with 


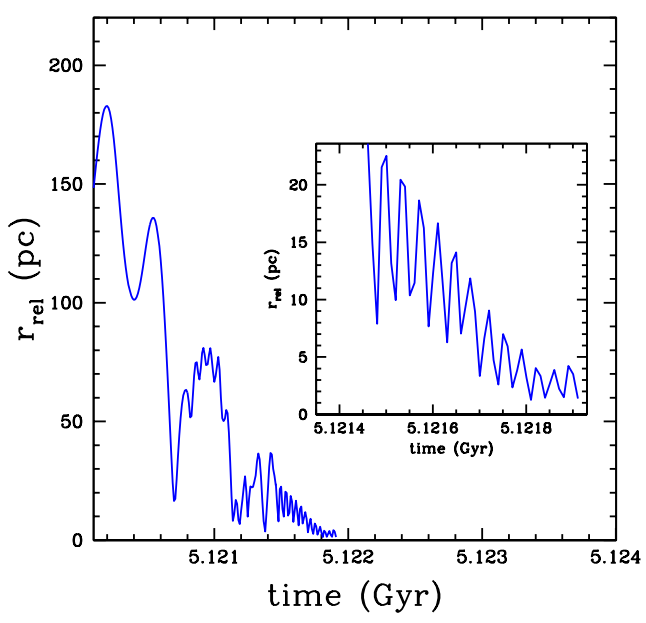

FIGURE 1. Relative separation of the two SMBHs as a function of time during the last stage of the standard, multi-scale merger simulation with $\gamma=7 / 5$. This value of $\gamma$ approximates well the balance between radiative heating and cooling in a starburst galaxy. The two peaks at scales of tens of parsecs at around $t=5.1213 \mathrm{Gyr}$ correspond to the end of the phase during which each black hole is still embedded in a distinct gaseous core. The inset shows the details of the last part of the orbital evolution, which takes place inside the nuclear disk arising from the merger of the two galactic cores. A SMBH binary forms rapidly, less than a million years after the coalescence of the two galactic nuclei, owing to the drag exerted by the surrounding dense gaseous nuclear disk.

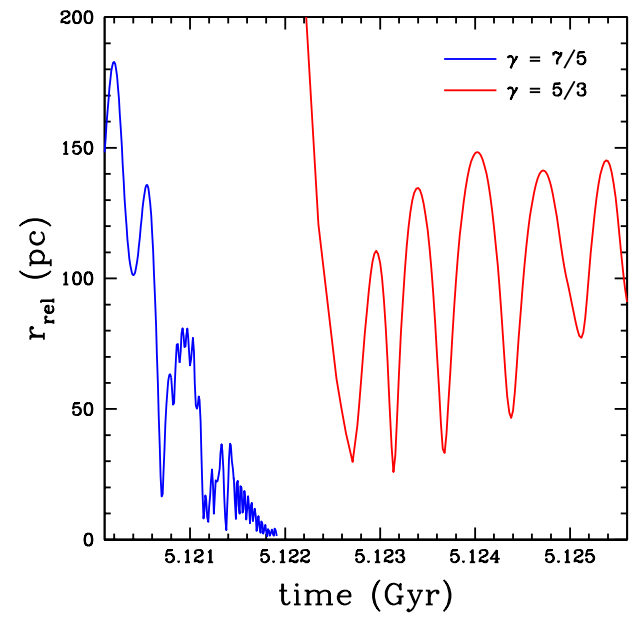

FIGURE 2. Relative separation of the two SMBHs as a function of time in two multi-scale, merger simulations with different prescriptions for the gas thermodynamics. The stiffer equation of state $(\gamma=5 / 3)$ corresponds to a situation where radiative cooling is completely suppressed by a strong heating source (e.g. AGN feedback) and causes the hardening process to significantly slow down. The orbital decay and pairing of SMBHs depends sensitively on the details of gas thermodynamics. 
a mass of $3 \times 10^{9} M_{\odot}$ and a size of $\sim 75$ pc. The two SMBHs are embedded in this nuclear disk. The disk is surrounded by several rings and by a more diffuse, rotationallysupported envelope extending out to more than a kiloparsec. A background of dark matter and stars distributed in a spheroid is also present but the gas component is dominant in mass within a few hundred pc from the center. From here on the orbital decay of the black holes is dominated by dynamical friction against this fairly dense gaseous disk. The black holes are on eccentric orbits and move with a speed of $v_{\mathrm{BH}} \sim$ $200-300 \mathrm{~km} / \mathrm{s}$ relative to the disk's center of mass. The typical ambient sound speed is $v_{s} \sim 50 \mathrm{~km} / \mathrm{s}$. The relative orbit of the SMBH pair decays from about $40 \mathrm{pc}$ to a few parsecs, our resolution limit, in less than a million years after the merger of the two galaxies (Figure 1). At this point the two black holes are gravitationally bound to each other, as the gas mass enclosed within their separation is less than the mass of the binary. Dynamical friction against the stellar background would bring the two black holes this close only on a much longer timescale, $\sim 3 \times 10^{7}$ yr (Section 4 ). Such a short sinking timescale due to the gas is expected because of the high densities in the nuclear disk and because the decay occurs in the supersonic regime with $v_{\mathrm{BH}}>v_{s}$ [25]. The subsequent hardening of the binary will depend on the details of gas dynamics and other processes at scales below the adopted resolution (Sections $6 \& 7$ ).

It is interesting to investigate the effect of the adopted equation of state on the orbital decay of the black holes. In particular, we considered an increased $\gamma$ to 5/3. This value of $\gamma$ would correspond to a purely adiabatic gas, or equivalently to a situation where radiative cooling is completely suppressed. The radiative feedback from an active galactic nucleus (AGN) is a good candidate for such a strong heating source. In this case, we find that a turbulent, pressure supported cloud of a few hundred parsecs arises from the merger rather than a disk. The nuclear region is still gas dominated, but the gas mass is lower within $100 \mathrm{pc}$ relative to the $\gamma=7 / 5$ case. This is because of the adiabatic expansion of the gas following the final shock when the two cores merged.

Figure 2 demonstrates that the hardening process is significantly suppressed when a stiffer equation of state with $\gamma=5 / 3$ is adopted. In this case, the black holes do not form a binary and maintain a relative separation of $\sim 100-150 \mathrm{pc}$ well after the binary forms in the simulation with $\gamma=7 / 5$. The density of the gas in the nuclear region surrounding the SMBHs is a factor of $\sim 5$ lower compared to that in the $\gamma=7 / 5$ case, the sound speed is $v_{s} \sim 100 \mathrm{~km} / \mathrm{s}$, and the black hole velocity is $v_{\mathrm{BH}}<100 \mathrm{~km} / \mathrm{s}$. The lower density and, to a lesser extent, the fact that the two black holes move subsonically $\left(v_{\mathrm{BH}}<v_{s}\right)$ rather than supersonically greatly reduce the drag due to the gas distribution when $\gamma=5 / 3$ [25]. In Section 5, we briefly discuss how the structure and kinematics of the nuclear regions of merger remnants in the simulations with different values of $\gamma$ compare to those of observed systems.

Given the sensitivity of the black hole pairing process to the value of $\gamma$, a scenario in which the black holes rapidly form a binary owing to dynamical friction against the gas would require that AGN feedback has negligible thermodynamical effects on small scales. In fact, this may be a more general requirement if the ubiquitous nuclear disk-like structures seen in many merger remnants are to be preserved. Interestingly, previous studies that included a prescription for AGN feedback in similar galaxy merger simulations [e.g. 26] find that feedback affects strongly the thermodynamics of the gas in the nuclear region only $>10^{8} \mathrm{yr}$ after the galaxy merger is completed. 
We have begun to carry out new adaptive mesh refinement (AMR) simulations using RAMSES [27], starting with orbits and galaxy models as close as possible to those used in the SPH simulations (Chapon, Teyssier \& Mayer, in preparation). The first results can be seen in Figure 3. The AMR simulations confirm the formation of bound binary with the $\gamma=7 / 5$ equations of state (EOS) and the stalling of the binary with the $\gamma=5 / 3$ EOS. The refinement capabilities of RAMSES allow to undertake more efficiently a study at increasingly higher resolution. The AMR structure is refined according to a density criterion. The AMR grid is divided in $2^{d}=8$ cells if its matter content exceeds a given mass of $m=m_{\text {refine }} \times 2 \times 10^{4} M_{\odot}$ (the main component of the nuclear disk is the gas). In the nuclear disk region $(\mathrm{r}<100 \mathrm{pc})$, the $m_{\text {refine }}$ parameter is set decreasingly from $8(\Delta x=25 p c)$ to $1(\Delta x=0.1 p c)$. This way, every AMR cell cannot contain more than $2 \times 10^{4} M_{\odot}$ of gas in the final nuclear disk.

As shown by Figure 3, as the spatial resolution improves beyond 1 parsec the decay speeds up and the binary hardens further, suggesting that the last parsec problem can be overcome in gaseous backgrounds. The faster decay at higher resolution is due to the fact that higher background densities can be resolved and do occur with increasing resolution. However, we also find that the hardening below a parsec scale might be sensitive to when additional refinements are applied. A new simulation carried out with GASOLINE and a resolution of $0.1 \mathrm{pc}$ shows a stalling of the binary below parsec scales (see section 5), and correspondingly has a disk which is more gravitationally unstable than its RAMSES counterpart. Overall, it appears clear that the sub-pc scale part of the decay of the binary SMBH is a difficult numerical problem since it is sensitive to the details of the internal disk dynamics, which in turn depend on resolution, thermodynamics and, possibly, code details.

\section{DYNAMICAL FRICTION TIMESCALES}

It is important to examine if the black holes could still form a binary as a result of the interaction with the collisionless stellar background. Since the resolution of the collisionless components is likely inadequate to assess directly the effect of dynamical friction (Section 2), we opt to calculate the dynamical friction timescale in the collsionless background analytically [28]

$$
\tau_{\mathrm{DF}}=1.2 \frac{V_{\mathrm{cir}} r_{\mathrm{cir}}^{2}}{G M_{\mathrm{BH}} \ln \left(M_{\mathrm{sd}} / M_{\mathrm{BH}}\right)} \varepsilon^{0.4} .
$$

Here $V_{\text {cir }}$ and $r_{\text {cir }}$ are, respectively, the initial orbital velocity and the radius of the circular orbit with the same energy as the actual orbit of the black holes, $\varepsilon$ is the circularity of the orbit, and $M_{\mathrm{sd}}$ is the sum of the dark matter and stellar mass within $r_{\text {cir }}$.

We calculate the decay time when the two black holes are separated by $100 \mathrm{pc}$, that is at the periphery of the nuclear disk just after the galaxy merger. Based on the simulations, we have $r_{\text {cir }}=100 \mathrm{pc}, V_{\text {circ }}=200 \mathrm{~km} / \mathrm{s}, \varepsilon=0.5, M_{\mathrm{BH}}=2.6 \times 10^{6} M_{\odot}$ and $M_{\mathrm{sd}}=5 \times 10^{8} M_{\odot}$. We find that the dynamical friction timescales in the collisionless background are equal to $5 \times 10^{7}$ yr and $3 \times 10^{7}$ yr in the $\gamma=5 / 3$ and $\gamma=7 / 5$ simulations, respectively (the shorter timescale in the $\gamma=7 / 5$ case is due to the fact that the stars and 
halo contract adiabatically more in response to the higher gas mass concentration in this case, and hence $M_{\text {sd }}$ is higher). In comparison, the binary formation timescale in the simulation with $\gamma=7 / 5$ was only $5 \times 10^{5} \mathrm{yr}$ (Figure 1 ).

We stress that Eq. (1) was derived for an isothermal sphere. The stellar and dark matter distribution are indeed only mildly triaxial within a few hundred parsecs from the center of the remnant and the total density profile is fairly close to $\rho(r) \propto r^{-2}$, as expected from previous work [e.g. 10].

We also compared our results with the expected dynamical friction timescale due to the gaseous background. In the simulation with $\gamma=7 / 5$, the gas is distributed in a disk rather than in an isothermal sphere. Since the disk thickness is $>10$ times the black hole gravitational softening and because of the fact that the density profile of the disk can be roughly approximated with a power law with an index close to 2 (except at the center where it becomes steeper) we are allowed to use Eq. (1) to obtain a rough estimate of the timescales. As shown by [9], analytical predictions with a fixed Coulomb logarithm [25] can overestimate the drag in the supersonic regime by a factor of $\sim 1.5$. In the $\gamma=7 / 5$ simulation, the black holes move supersonically and the analytical formula should yield the correct prediction. In this case the drag is a factor of $\sim 2.3$ stronger than in the corresponding collisionless case [9]. This is fairly consistent with our results. Indeed, eq. (1) with a reduction of a factor of 2.3 gives $\sim 10^{6} \mathrm{yr}$ if we set $M_{\text {gas }}=M_{\text {sd }}$, with $M_{\text {gas }} \sim 20 M_{\text {stars. }}$. This timescale has to be compared with that measured directly in the simulation, $5 \times 10^{5} \mathrm{yr}$. As discussed above, the gas profile is actually steeper than $r^{-2}$ near the center. Thus, it is not surprising that the decay is faster. Despite the apparent agreement with the analytically estimated drag, we note that the orbital evolution of the two black holes might be affected by more than just the gravitational wake. Indeed, the nuclear disks show strong, highly dynamical non-axisymmetric structures such as spiral arms (see Section 7) which are highly efficient at removing angular momentum from the orbiting SMBHs.

The drag drops rapidly by an order of magnitude in the subsonic regime [9]. This coupled with the fact that $M_{\text {gas }}$ is a factor of $\sim 5$ lower in the simulation with $\gamma=5 / 3$ compared to that with $\gamma=7 / 5$ would give a drag 50 times smaller or $\tau_{\mathrm{DF}} \sim 5 \times 10^{7} \mathrm{yr}$, explaining why the orbital decay caused by the gas is so inefficient in this case. Thus, in the $\gamma=5 / 3$ simulation stars and gas contribute to the drag in a comparable way.

\section{FORMATION OF SUPERMASSIVE BLACK HOLES}

The formation of SMBHs themselves is still an open problem. Conventional models, which assume that SMBHs grow from seeds of a few hundred solar masses formed by the collapse of Pop III stars [16], cannot explain the rapid emergence of bright QSOs at $z>6$ revealed by the Sloan Digital Sky Survey (SDSS) [15]. Previous models that can form the $10^{9} M_{\odot}$ black holes that power such QSOs in the available time need to start from seeds having a mass of $10^{5} M_{\odot}$ already at $z=10$ [29], which cannot be achieved starting from collapsing Pop III stars due to inefficient gas accretion [30, 31, 32]. An alternative is to postulate that massive seeds form by direct collapse of large quantities of gas, without prior formation of a star. Gravitational torques in gas-rich protogalaxies, either via spiral instabilities [33] or via bars-in-bars instabilities [34, 35] can transport 
efficiently angular momentum outwards and gas inwards. The first direct simulations of protogalactic evolution at $z>10$ confirm that more than a million solar mass can be accumulated within the central parsec $[36,37]$. However, the ultimate fate of such gas is unknown. Even more importantly, these simulations assume gas of primordial composition which cannot cool below $10^{4} \mathrm{~K}$, and therefore cannot fragment into stars. Fragmentation and star formation would reduce the amount of gas available for the formation of a massive SMBH seed, and could change the dynamics of the central region, interfering with the large-scale global torques responsible for efficient mass and angular momentum transfer. Assuming metal-free conditions in protogalaxies is highly idealized since as soon as the first generation of stars produces metals, cooling below $10^{4}$ $\mathrm{K}$ would become important, even at very low metallicities if dust grain formation occurs [38]. Therefore, whether instabilities in protogalaxies are the route to form massive black hole seeds is still unclear.

Another possibility is to consider galaxy mergers, that are known to provide the strong angular momentum losses on galactic scales, and to transport more than half of the gas from kiloparsec scales to scales of hundred parsecs, triggering major starbursts $[21,10]$. Whether large quantities of gas can be funnelled down to sub-pc scales and trigger the formation of a massive seed SMBH had not been explored until very recently. In [39] we performed multi-scale major merger simulations similar to those described above, using the same initial galaxy models and orbits but without including a SMBH, and reaching a spatial resolution an order of magnitude better (the gravitational softening after particle splitting is $0.1 \mathrm{pc}$ ). The merger product of these two Milky-Way sized galaxies has a total mass of $\sim 2 \times 10^{12} M_{\odot}$, which is compatible with the low end of the possible host halo masses of the bright SDSS QSOs at $z>6$ based on clustering statistics. At such redshifts this halo mass corresponds to that of a highly biased object, for which it is sensible to assume that it was built via one or more nearly equal mass mergers [29]. The structure of protogalactic disks at these redshifts is not known yet, but the latest numerical simulations suggest that rotationally supported gas-rich disks not unlike those of our galaxy models should form already between $z=15$ and $z=5$ within relatively massive halos $[37,35]$. We adopt the same EOS model of the [18] work but this time including a switch to a lower value of the adiabatic exponent, $\gamma=1.1$, as the density rises above $10^{6}$ atoms $/ \mathrm{cm}^{3}$ (such densities were barely resolved in the [18] work that was adopting a coarser spatial resolution corresponding to $2 \mathrm{pc}$ ).

As soon as the two galaxy cores merge the nuclear disk assembles in an unstable state, with a prominent $m=2$ mode imprinted. The spiral instability drives mass inward and angular momentum outwards on a timescale comparable to the disk orbital time, which is less than $10^{5}$ years at its scale size of nearly a hundred parsec. The gas inflow rate within the nuclear disk peaks at $10^{5} M_{\odot} / \mathrm{yr}$ and averages at $10^{3} M_{\odot} / \mathrm{yr}$. In less than $10^{5} \mathrm{yr}$ several times $10^{8} M_{\odot}$ of gas accumulate within the central few parsecs, and as the EOS becomes softer the mass concentration climbs above the local Jeans mass at a scale of about a parsec, collapsing into a hot, pressure-supported supermassive cloud whose further contraction is only stopped by our limited resolution. The supermassive cloud is the likely precursor of a SMBH. Its high temperature $\left(T>10^{7} \mathrm{~K}\right)$ implies that fragmentation into stars cannot occur within it, hence it is will either form a supermassive star and later collapse into a SMBH seed via the post-newtonian radial instability [40] or form a lowentropy, quasi-star which can generate a massive SMBH seed weighting $>10^{5} M_{\odot}$ via 
rapid super-Eddington accretion onto an initial seed of a few tens of solar masses formed at its center [41]. In either case the seed would form on a timescale shorter than the disks'

orbital time of $10^{5} \mathrm{yr}$, and can easily accrete from the huge reservoir of molecular gas in the disk ( $>10^{9} M_{\odot}$ ) (in contrast with Pop III seeds models, in which the surrounding gas does not accrete efficiently because it is hot and ionized), reaching a billion solar mass in less than $3 \times 10^{8} \mathrm{yr}$ assuming Eddington-limited accretion for a radiative efficiency of about $10 \%$ [40]. The timescale of a typical galaxy merger occurring at $z \sim 8$ is itself a few times $10^{8} \mathrm{yr}$, hence overall less than a billion years would be required to reach the $\sim 10^{9} M_{\odot}$ estimated for the SDSS QSOs, thus matching the observations.

The nuclear disk, while violently spirally unstable, does not fragment. The stiff EOS implied by the presence of the starburst (see section 2) stabilizes the disk against global fragmentation, enforcing a Toomre $Q$ parameter larger than unity everywhere. Therefore global fragmentation is not an issue for the gas inflow in our model. Star formation is expected to reach several thousand solar masses per year in the densest regions of the disk if we simply assume a Kennicutt-Schmidt law, an impressive rate consistent with what is found in cosmological simulations of massive galaxy assembly via mergers at $z>5$ [29] but still much lower than the peak inflow rate of $10^{5} M_{\odot} / y$, another indication that the starburst and the SMBH-forming inflow can easily co-exist. Star formation of course implies fragmentation, but local rather than global; local, small scale fragmentation cannot be modeled by our EOS scheme, which misses a description of the star forming molecular phase since by construction it assumes a one-phase model whose properties, including the Toomre parameter, are a volume-average of those of the individual phases in a realistic multi-phase ISM ( see section 2). New simulations in progress that include star formation and include radiative cooling do confirm that a prominent gas inflow accumulating more than $10^{8} M_{\odot}$ of gas in the central few parsecs occurs even when fragmentation occurs locally in the nuclear disk. Indeed this result is not surprising at all; simulations of gravitational instabiity in another context, giant planet formation in protoplanetary disks, had already shown that gas inflows, indeed the most prominent among them, do occur in disks that undergo local fragmentation into clumps [42].

\section{THE INTERPLAY BETWEEN FORMATION AND ORBITAL DECAY OF SMBHS}

The possible formation scenario described in the previous sections has interesting consequences on the last parsec problem. In principle the rapid and prominent gas inflow described above could take place even if a SMBH is already present at the center of the merging galaxies. Milky-Way sized disk galaxies, while fairly high-sigma peaks at $z>5$, are indeed increasingly more common towards lower redshifts, when a central SMBH would have been formed already. How would the decay below parsec scales proceed in such conditions? In order to address this issue we have rerun the major merger simulation with $0.1 \mathrm{pc}$ gravitational softening described in the previous section adding a central SMBH at the center of the two galaxies as explained in section 2. With increased resolution two black holes sink somewhat faster than in the [18] as in the AMR simulations with deeper refinements (see end of section 3 and Figure 3). By the time the two 


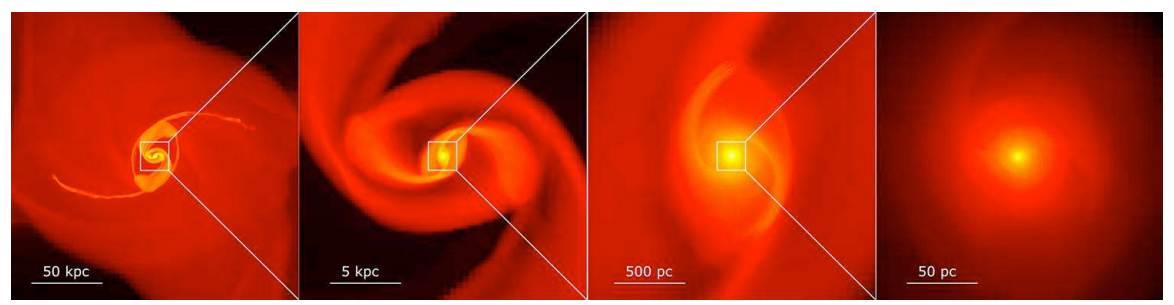

FIGURE 3. Projected density maps of the nuclear disk viewed face-on in an AMR numerical simulation with $\gamma=7 / 5$ and $3.4 \mathrm{pc}$ spatial resolution in the gas. Panels display the nuclear disk $\sim 10^{6} \mathrm{yr}$ after the galaxy merger is deemed complete, with different zoom-in factors.

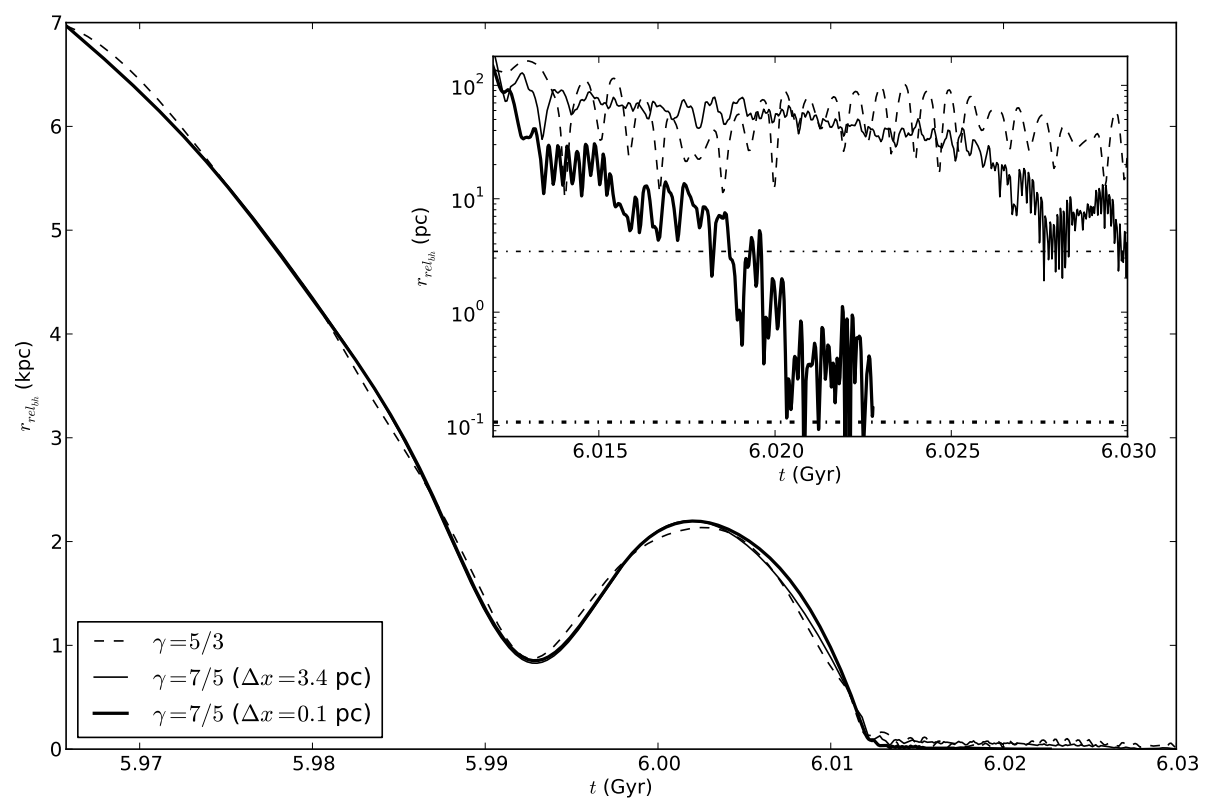

FIGURE 4. Orbital evolution of the two SMBHs as a function of time in AMR simulations with different resolutions and varying adiabatic index $\gamma$ (see labels in the figure).The resolution limits are shown in dash-dotted lines of the corresponding line style. While the relative separation of the two black holes stabilizes around $60-80 \mathrm{pc}$ in the $\gamma=5 / 3$ run, their orbital decay decreases down to the resolution limit in the $\gamma=7 / 5$ runs. This confirms the results of the SPH runs.

black holes reach a separation of about $1 \mathrm{pc}$ the central supermassive cloud has already formed, sweeping most of the mass from the inner disk. As a result of the central inflow, the density of the gas outside the central parsec decreases by a factor of $\sim 5$. The density reduction weakens the effect of dynamical friction. In addition, because the two black 


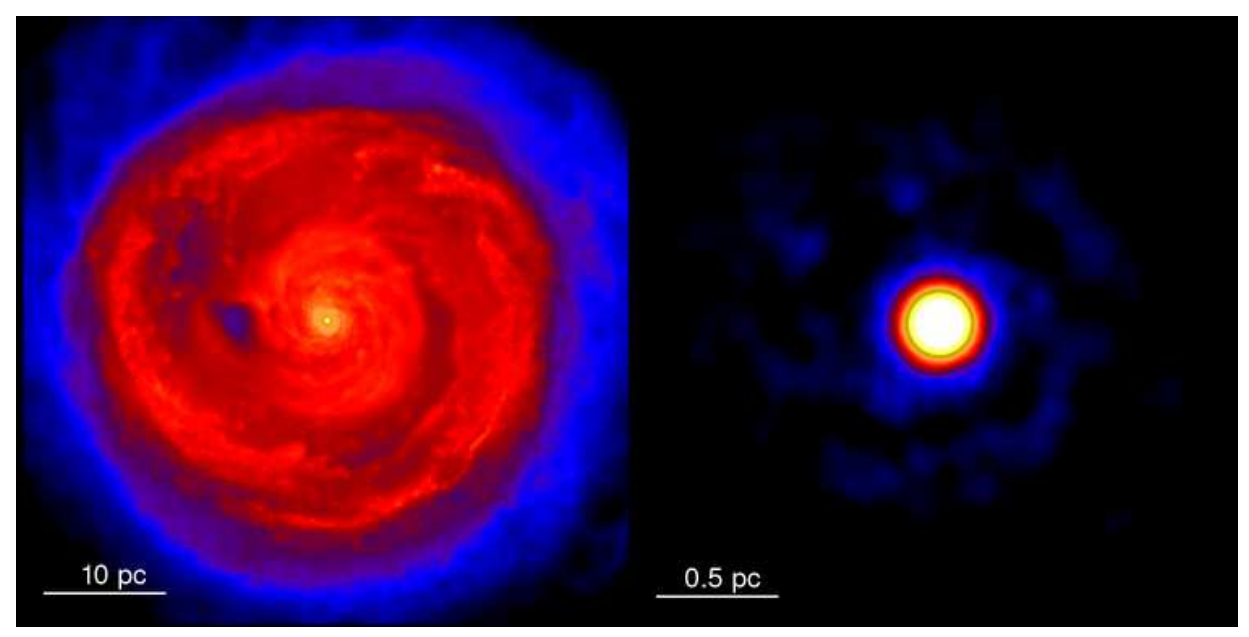

FIGURE 5. Color-coded projected density maps of the nuclear disk viewed face-on in the numerical simulation with $0.1 \mathrm{pc}$ spatial resolution in the gas. Both panels display the nuclear disk $\sim 10^{5} \mathrm{yr}$ after the galaxy merger is deemed complete. The left panel shows the large scale structure of the disk, with evident distortions and spiral pattern. The right panel presents the inner region of the disk. A conspicuous central cloud, which is Jeans-unstable, is present. This is a possible precursor of a massive SMBH seed.

holes are now orbiting around the central cloud, their relative velocity is a factor of $\sim 3$ higher compared to that in the standard $2 \mathrm{pc}$ simulation in which the central clump was not resolved. The dynamical friction force scales as $\rho / v_{\mathrm{BH}}^{2}$. The combined reduction of $\rho$ and increase of $v_{\mathrm{BH}}$ results in an overall decrease of almost a factor of 50 in the strength of dynamical friction, explaining the observed suppression in the orbital decay by almost two orders of magnitude. Moreover, as a result of the clump formation, the mass contained within the orbit of the two black holes has become much larger than the sum of their masses; the two black holes not only stall at a separation just below a parsec but also form only a loose pair rather than a binary as in [18].

How should we interpret the suppression of the orbital decay in presence of the massive central cloud formed by the gas infow? Gas inflows are expected in nonaxisymmetric disks, and they are indeed reported in high-resolution simulations of nuclear disks starting from equilibrium conditions [43, 44, 45], and also in the large set of multi-stage simulations of galaxy mergers recently carried out by [46]. Yet the magnitude of the inflows, hence the mass of the central clump and associated variation of the disk density profile, is somehow exaggerated by the crude modeling of the ISM, and by the lack of star formation, supernovae feedback, and gas accretion onto the SMBHs. Even more important, if the SMBHs are already present they would likely accrete and produce energetic feedback onto the surrounding disk before they reach a separation of parsecs since there is a large gas reservoir in the nuclear disk even at its outskirt, at scales of tens of parsecs. This additional heating could serve to heat the nuclear gas and stabilize the disk by rising its Toomre Q, weakening the inflow and allowing the SMBHs to sink efficiently below parsec-scale separations. That a slightly hotter, more 
stable disk would lead to efficient hardening of the binary is suggested by the AMR results shown in Figure 3. In those simulations, since the highest level of refinement is applied only after the merger, the inflow is smoothed out - not enough mass accumulates to produce the central supermassive cloud and, as expected, the decay continues down to the resolution limit of $0.1 \mathrm{pc}$. While this result is driven by the lack of resolution, a physical heating source capable of increasing the stability of the disk and weakening the inflow would achieve the same result. Therefore we are facing the following intriguing scenario; SMBHs may form by direct collapse in gas-righ mergers at high-z, but once they do so their energetic feedback in subsequent mergers at the same time prevents the formation of a new massive SMBH seed at the center of the merger remnant and allows the two holes to bind into a binary, harden and finally coalesce. This could be a new important self-regulation mechanism driving the co-evolution of the nuclear regions of galaxies and supermassive black holes through cosmic time.

\section{REFERENCES}

1. Ferrarese, L., Ford, H., SSRv 116, 523 (2005)

2. Blumenthal, G. R., Faber, S. M., Primack, J. R., Rees, M. J., Nature 311, 517 (1984)

3. Begelman, M. C., Blandford, R. D., Rees, M. J., Nature 287, 307 (1980)

4. Milosavljević, M., Merritt, D., ApJ 563, 34 (2001)

5. Vecchio, A., Phys. Rev. D 70, 042001 (2004)

6. Berczik, P., Merritt, D., Spurzem, R., ApJ 633, 680 (2005)

7. Downes, D., Solomon, P. M., ApJ 507, 615 (1998)

8. Davies, R. I., Tacconi, L. J., Genzel, R., ApJ 613, 781 (2004)

9. Escala, A., Larson, R. B., Coppi, P. S., Mardones, D., ApJ 607, 765 (2004)

10. Kazantzidis, S., Mayer, L., Colpi, M., et al., ApJL 623, L67 (2005)

11. Escala, A., Larson, R. B., Coppi, P. S., Mardones, D., ApJ 630, 152 (2005)

12. Dotti, M. Colpi, M. Haardt, F., MNRAS 367, 103 (2006)

13. Dotti, M., Colpi, M., Haardt, F., Mayer, L., MNRAS 379, 956 (2007)

14. Sesana, A., Volonteri, M., Haardt, F., MNRAS 377, 1711 (2007)

15. Fan, X., NewAR 50, 665 (2006)

16. Madau, P., Rees, M. J., ApJL 551, L27 (2001)

17. Volonteri, M., Rees, M. J., ApJ 650, 669 (2006)

18. Mayer, L., Kazantzidis, S., Madau, P., et al., Science 316, 1874 (2007)

19. Klypin, A., Zhao, H., Somerville, R. S., ApJ 573, 597 (2002)

20. Khochfar, S., Burkert, A., $A \& A$ 445, 403 (2006)

21. Barnes, J. E., Hernquist, L., ApJ 471, 115(1996)

22. Bate, M. R., Burkert, A., MNRAS 288, 1060 (1997)

23. Wadsley, J. W., Stadel, J., Quinn, T., NewA 9, 137 (2004)

24. Spaans, M., Silk, J., ApJ 538, 115 (2000)

25. Ostriker, E. C., ApJ 513, 252 (1999)

26. Springel, V., Di Matteo, T., Hernquist, L., MNRAS 361, 776 (2005)

27. Teyssier, R., A\&A 385, 337 (2002)

28. Colpi, M., Mayer, L., Governato, F., ApJ 525, 720 (1999)

29. Li, Y., Hernquist, L., Robertson, B., Cox, T. J., et al., ApJ 665, 187 (2007)

30. Johnson, J. L., Bromm, V., MNRAS 374, 1557 (2007)

31. Alvarez, M. A., Wise, J. H., Abel, T., ApJL 701, L133 (2009)

32. Milosavljević, M., Couch, S. M., Bromm, V., ApJL 696, L146 (2009)

33. Lodato, G., Natarajan, P., MNRAS 371, 1813 (2006)

34. Shlosman, I., Frank, J., Begelman, M. C., Nature 338, 45 (1989)

35. Levine, R., Gnedin, N. Y., Hamilton, A. J. S., Kravtsov, A. V., ApJ 678, 154 (2008)

36. Wise, J. H., Turk, M. J., Abel, T., ApJ 682, 745 (2008) 
37. Regan, J. A., Haehnelt, M. G., MNRAS 396, 343 (2009)

38. Omukai, K. Schneider, R., Haiman, Z., ApJ 686, 801 (2008)

39. Mayer, L., Kazantzidis, S., Escala, A., Callegari, S., arXiv:0912.4262 (2009)

40. Shapiro, S. L., ApJ 620, 59 (2005)

41. Begelman, M. C., Volonteri, M., Rees, M. J., MNRAS 370, 289 (2006)

42. Mayer, L., Quinn, T., Wadsley, J., Stadel, J., ApJ 609, 1045 (2004)

43. Escala, A., ApJL 648, L13 (2006)

44. Escala, A., ApJ 671, 1264 (2007)

45. Kawakatu, N., Wada, K., ApJ 681, 73 (2008)

46. Hopkins, P. F., Quataert, E., arXiv:0912.3257 (2009) 